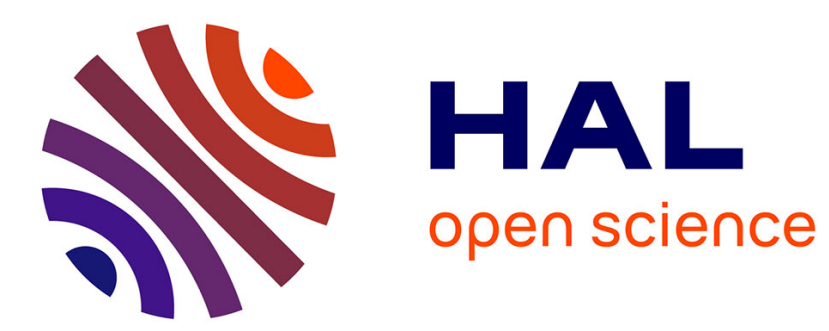

\title{
A squeezing experiment showing binder migration in concentrated suspensions
}

Arnaud Poitou, Guillaume Racineux

\section{To cite this version:}

Arnaud Poitou, Guillaume Racineux. A squeezing experiment showing binder migration in concentrated suspensions. Journal of Rheology, 2001, 45 (3), pp.609-625. 10.1122/1.1366717 . hal01004947

\section{HAL Id: hal-01004947 \\ https://hal.science/hal-01004947}

Submitted on 28 Jan 2017

HAL is a multi-disciplinary open access archive for the deposit and dissemination of scientific research documents, whether they are published or not. The documents may come from teaching and research institutions in France or abroad, or from public or private research centers.
L'archive ouverte pluridisciplinaire HAL, est destinée au dépôt et à la diffusion de documents scientifiques de niveau recherche, publiés ou non, émanant des établissements d'enseignement et de recherche français ou étrangers, des laboratoires publics ou privés. 


\title{
A squeezing experiment showing binder migration in concentrated suspensions
}

\author{
Arnaud Poitou \\ Laboratoire de Mécanique et Technologie, E.N.S. de Cachan, C.N.R.S., U.P.M.C., \\ 61 avenue du Président Wilson, 94235 Cachan Cedex, France \\ Guillaume Racineux \\ Centre de Mécanique des Milieux Continus, Université de Versailles Saint- \\ Quentin-en-Yvelines, 45 avenue des Etats Unis, 78035 Versailles Cedex, France
}

This paper deals with concentrated suspensions (pastes) of $\mathrm{T}_{i} \mathrm{O}_{2}$ particles at a solid concentration of $60 \%$ per weight in an acidic binder. This paste is used to manufacture petrochemical catalysts. First we show that in a squeezing experiment binder migration occurs in the suspension as long as the velocity at which the test is carried out does not exceed a critical value. Next, we develop a mathematical model of the test and a complete methodology to identify the rheological parameters of this model. We give an analytical expression of the critical velocity, which is related to the physical characteristics of the suspension. The exact identification of the rheological parameters requires the use of a numerical program which was developed for this application.

\section{INTRODUCTION}

Flow-induced migration of particles has been an active research field for many years. Acrivos' model [Leighton and Acrivos (1987); Phillips et al. (1992)] states that a particle placed in a flow which presents a strain gradient receives from the other particles, on the average, more shocks on the side of the flow that has the higher shear rate and, therefore, tends to migrate in the opposite direction. Acrivos' model consists of representing this phenomenon with a diffusion equation. Thus, it is possible to describe a Couette device experiment or a Poiseuille flow. More recently, these results were extended to three dimension by numerical methods. However, since Biot's studies, other models have been developed which are closer to soil mechanics theories [Bowen et al. (1976); Lantéri et al. (1991); Lantéri et al. (1996a); Dutilly (1998); Louge (1996); Casella (1997)]. Nevertheless, regardless of the model, the main difficulty in these approaches is to establish an experimental methodology which allows one to identify the rheological parameters on industrial materials. The aim of this paper is to present a solution to this problem. First, we will show that in a squeezing experiment particle migration-or, more precisely, binder migration - occurs as long as the velocity at which the test is carried out does not 


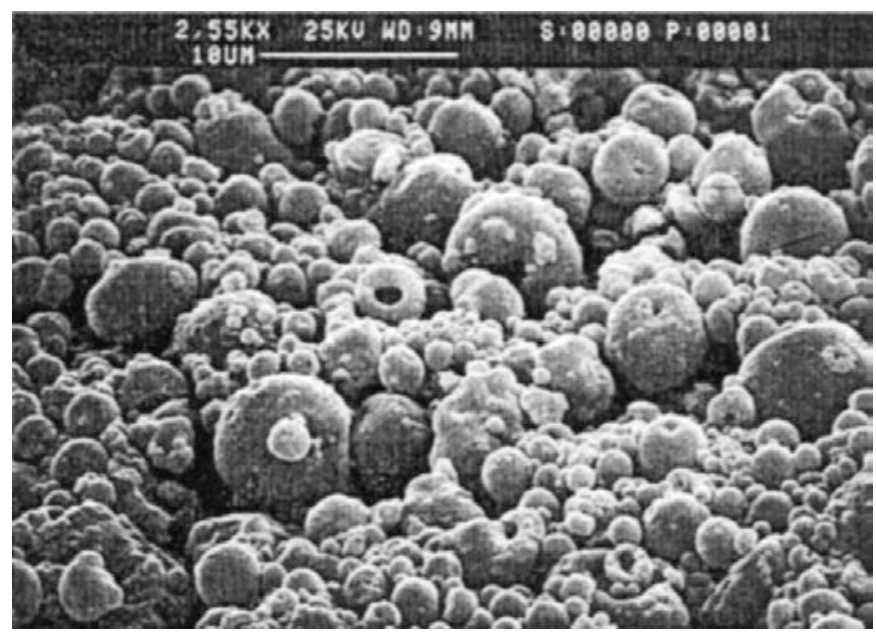

FIG. 1. SEM photomicrograph of $\mathrm{T}_{i} \mathrm{O}_{2}$ powder [Jabbes (1995)].

exceed a critical value. Next, we will develop a mathematical model to describe the test as well as a complete methodology to identify the rheological parameters of this model. We will give an analytical expression of the critical velocity, which is related to the physical characteristics of the suspension. The exact identification of the parameters requires the use of a numerical program, which was developed specifically.

\section{EXPERIMENTS}

\section{A. Materials}

The concentrated suspension used in this study was a paste at a solid concentration of $60 \%$ per weight. The Procatalyse Company produces this saturated paste by extrusion in order to manufacture petrochemical catalysts. The $\mathrm{T}_{i} \mathrm{O}_{2}$ powder is made of polydisperse grains with an average size of $4 \mu \mathrm{m}$. The powder is obtained by sol atomization, which causes the grains to be constituted of agglomerates of finer particles. The sol-gel process results in calibrated particles which are, on the average, about $40 \mathrm{~nm}$ in diameter. These elementary spheres themselves are aggregates of anastase microcrystallites of about $65 \AA$ [Jabbes (1995)]. Figure 1, is a typical scanning electron microscope photomicrograph which shows the morphology of the powder and the agglomerate size distribution. The different types of agglomerates can be seen: some have a diameter between 6 and $7 \mu \mathrm{m}$ while most of the others are around $1 \mu \mathrm{m}$. Their shape is approximately spherical. The specific mass of the powder is $3.35 \mathrm{~g} / \mathrm{cm}^{3}$ and its specific area is $280 \mathrm{~m}^{2} / \mathrm{g}$.

The binder is an aqueous $\left(\eta_{f}=10^{-3} \mathrm{~Pa} \mathrm{~s}\right)$ solution of concentrated nitric acid which acts as a peptization agent. Because of capillary forces, the wet powder is initially agglomerated and aggregated. The acid induces repulsive interactions among the aggregates: the particles are electrostatically charged by protons through a phenomenon similar to the D.L.V.O. mechanism. This colloidal action combined with mechanical mixing allows the agglomerates to break up, which leads to a homogeneous paste.

The mixing device is a Z-blade internal mixer. These blades scrape the paste against the recipient walls with a complex trajectory. Thus, the paste is subjected to a history of high and almost homogeneous shear. Its behavior is very sensitive to the water content; therefore, it is very important to conduct the mixing process carefully in order to obtain reproducible results:

(i) The mixer is cooled to avoid water losses by evaporation.

(ii) The powder is introduced first in order to avoid fluid flow through interstices located at the point where the blades enter the mixing recipient. 


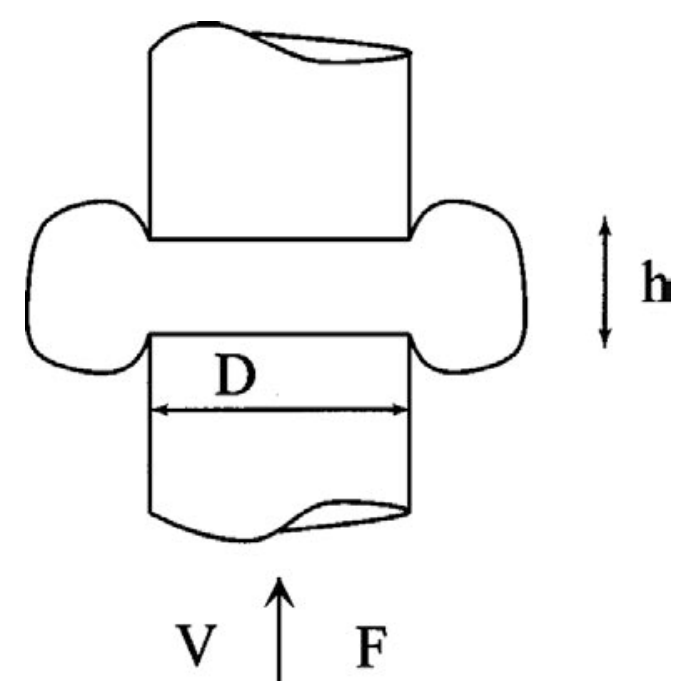

FIG. 2. Force vs gap size.

(iii) The dry powder is mixed a few minutes in order to break apart the largest agglomerates.

(iv) The binder is introduced in one step.

(v) The paste is then mixed for approximately $1 \mathrm{~h}$ to get a homogeneous product.

To control the agglomerate size after mixing, mercury porosimetry experiments were carried out on dry samples (i.e., without binder). The porosity of the skeleton is perfectly stable from one experiment to the next (void volume of $0.35 \mathrm{~cm}^{3} / \mathrm{g}$ and pore size between 10 and $100 \mathrm{~nm}$ ).

\section{B. Rheological measurement}

Squeezing tests were carried out on an M.T.S. servocontrolled hydraulic tension test machine. The squeezing device consists of two coaxial circular plates $(40 \mathrm{~mm}$ in diameter). The upper plate is fixed whereas the lower one moves vertically at a prescribed velocity $V$. The evolution of the squeezing force $F$ is measured against the size $h$ of the gap between the plates (see Fig. 2). The paste sample is placed between the plates and then squeezed at constant velocity. The initial gap $h_{0}$ between the two plates at the beginning of the tests is such that $h_{0} / D \ll 1$, where $D$ is the plate diameter.

\section{Initial conditions}

To ensure reproducible initial conditions, we prepared specimens with a constant initial thickness. Our procedure consisted of preforming them quickly in moulds with calibrated diameter $D$ and thickness $h_{0}$ (Fig. 3). The preshaped sample was then placed on the lower plate, which was then moved upwards until the sample reached the upper plate (Fig. 4).

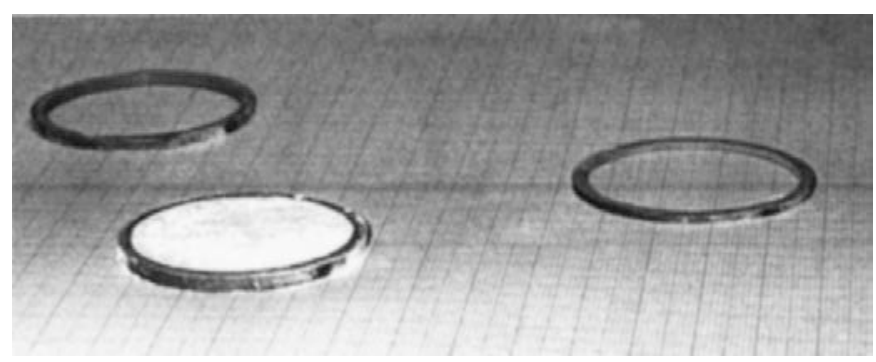

FIG. 3. The samples are preformed in calibrated moulds. 


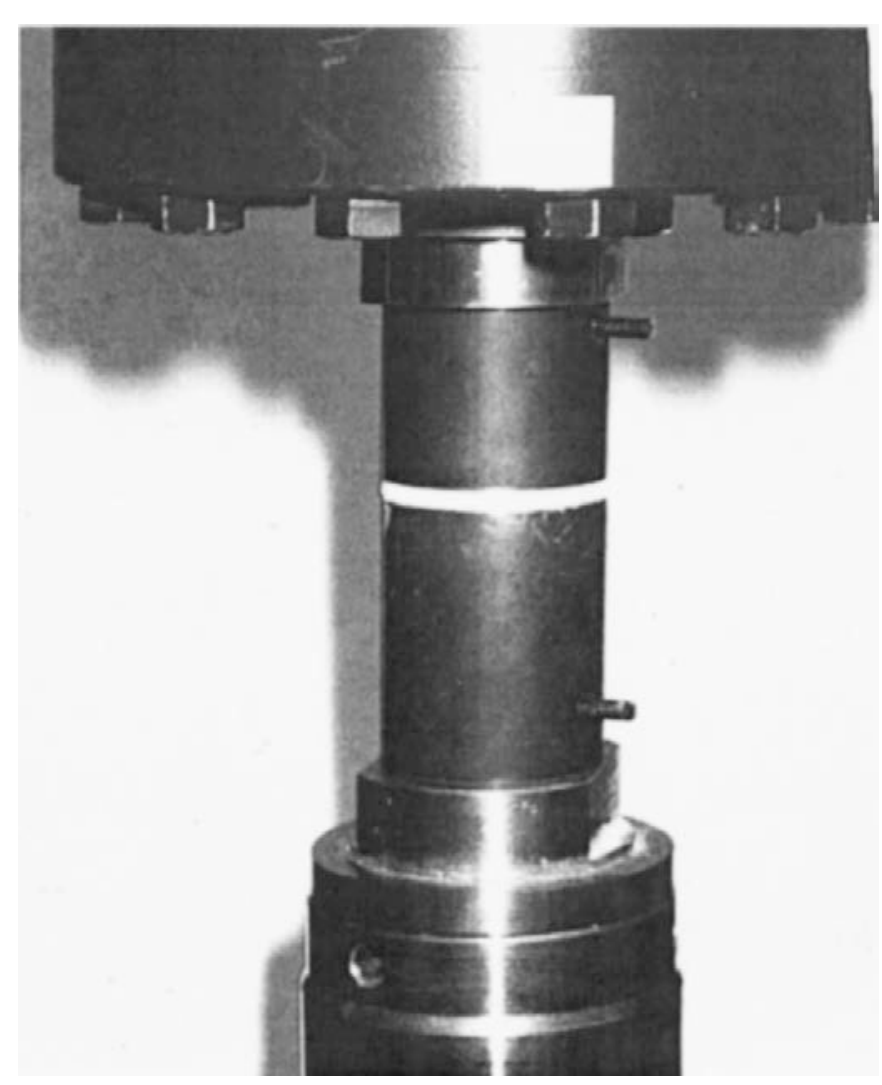

FIG. 4. Samples are placed on the lower plate, which is moved upwards until it reaches the upper plate.

\section{Boundary conditions}

To avoid slipping of the paste at the wall, we used roughened plates, i.e., plates onto which we pasted abrasive papers in order to force sticking contact at the wall. We used self-adhesive papers intended for surface polishing because they present the following advantages:

(i) Their grain size is controlled.

(ii) Polishing papers resist shearing very well.

(iii) Self-adhesive papers have good resistance to water, which means that they do not alter the composition of the paste during the test.

To confirm that the paste sticks on plates with abrasive papers, we tested plates with different surface roughness: polished plates made of rectified iron $(R a=0.4 \mu \mathrm{m})$ and plates with abrasive papers of different grain sizes: P400 (35 $\mu \mathrm{m})$, P600 $(26 \mu \mathrm{m})$, and P800 $(22 \mu \mathrm{m})$. Figure 5 shows the results of the tests with an initial gap size $h_{0}$ $=2.5 \mathrm{~mm}$ and piston velocity $V=0.02 \mathrm{~mm} / \mathrm{s}$ without abrasive paper and with P400, P600, and P800 abrasive papers. Two conclusions can be reached from these curves:

(i) The tests carried out with different kinds of abrasive papers give the same results: the curves obtained with the different papers are superimposed on Fig. 5.

(ii) The test carried out with polished plates (i.e., without paper) gives different results, indicating an interaction between the paste and the boundary. In this paper, we are interested only in tests with no slipping at the walls (i.e., with abrasive papers). 


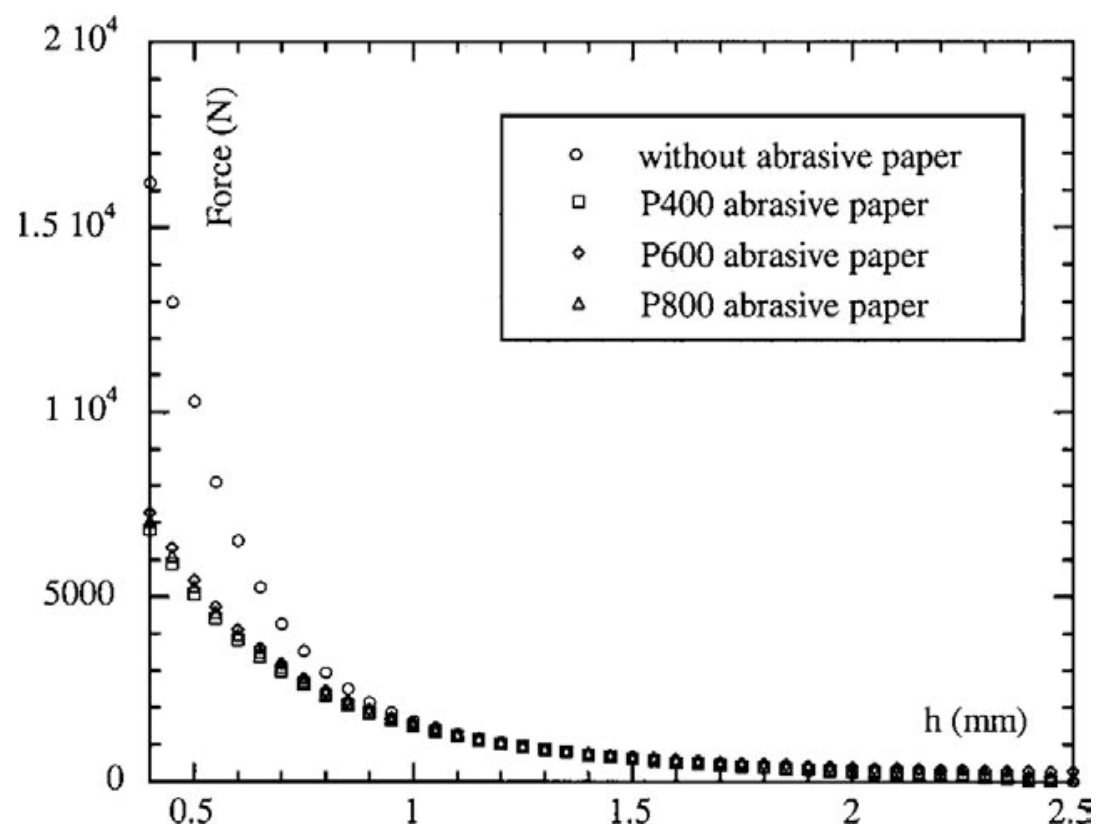

FIG. 5. Tests with P800 abrasive papers (initial gap $h_{0}=2.5 \mathrm{~mm}$ ).

\section{RESULTS AND DISCUSSION}

\section{A. Effect of velocity: dissociation}

Figure 6 shows the squeezing force curve at different squeezing velocities and with abrasive papers (i.e., with sticking contact at the wall). Figure 7 shows the force as a function of velocity for a few fixed gap widths. These results show effects which appear contradictory:

(i) For a squeezing velocity greater than a critical piston velocity $V_{c} \simeq 1 \mathrm{~mm} / \mathrm{s}$, the force increases with the velocity, but less than linearly (i.e., the force is less than doubled if the velocity is doubled).

(ii) On the contrary, for a squeezing velocity less than about $1 \mathrm{~mm} / \mathrm{s}$, the force increases when the velocity decreases.

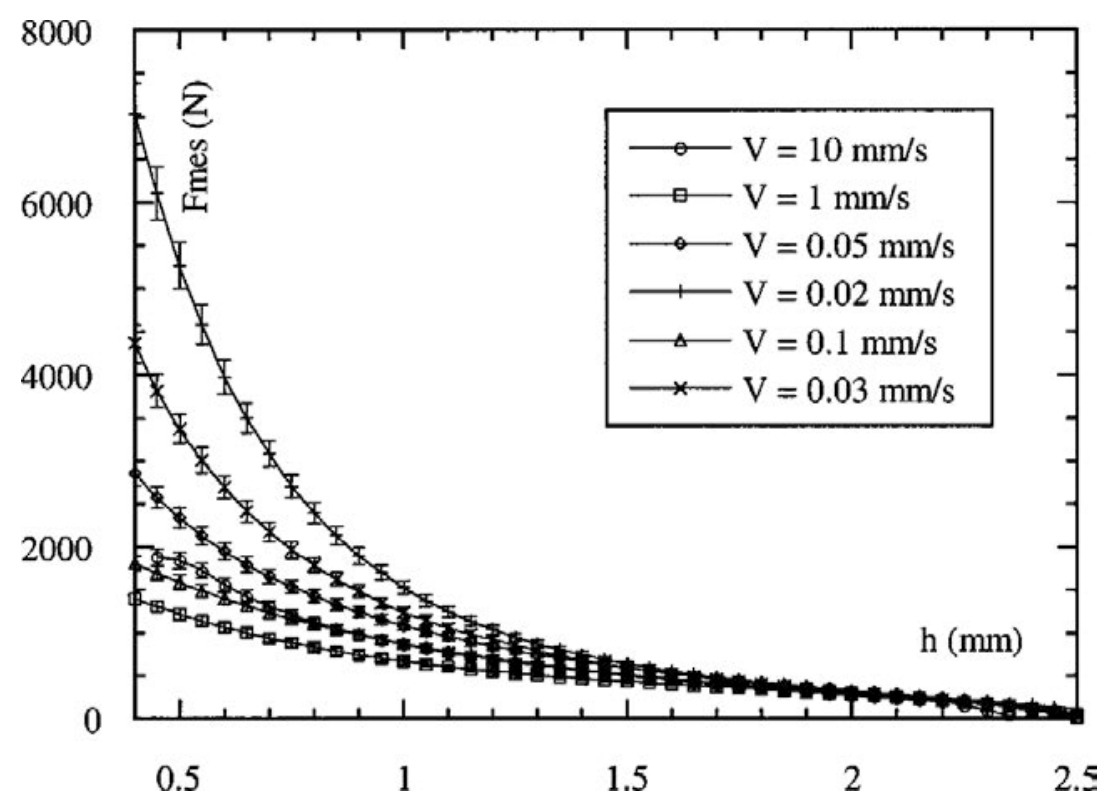

FIG. 6. Results of squeezing tests with initial gap $h_{0}=2.5 \mathrm{~mm}$ and abrasive papers. 


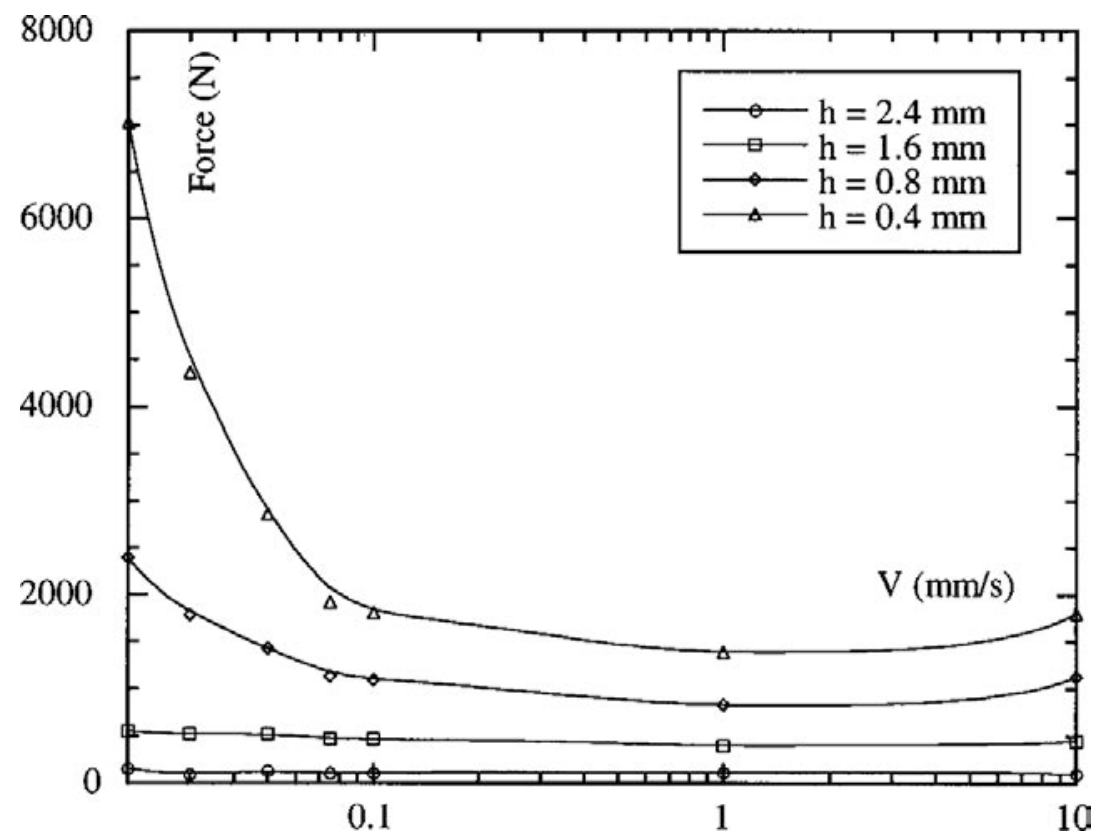

FIG. 7. Force as a function of velocity for a few fixed gap widths.

At the end of a test, the sample is very fine $(0.2 \mathrm{~mm})$ and it tears when one lifts the upper plate (Fig. 8). It was, therefore, impossible to take samples of paste in different places (at the center, on the edges...) with sufficient precision to measure the volume fraction of powder in the sample. Furthermore, in very fine samples, the binder evaporates very quickly. But after tests at low squeezing velocities, if we touched the sample we noted (at least qualitatively) that the paste remaining between the plates contains a higher volume fraction of powder at the end of the test than at the beginning and a higher volume fraction of powder in the center of plates than on the edges. This observation enables us to find an explanation for why the squeezing force at low velocities can be greater than at high velocities. A test carried out at low velocity is a drained test (the binder has had time to filter through the powder); therefore, at the end of the test, one measures the resistance of a quasicompact skeleton of powder. On the contrary, at higher velocity, the binder cannot flow through the powder and, thus, it behaves as a lubricant between the grains. The test results presented in Fig. 9 confirm qualitatively this drainage

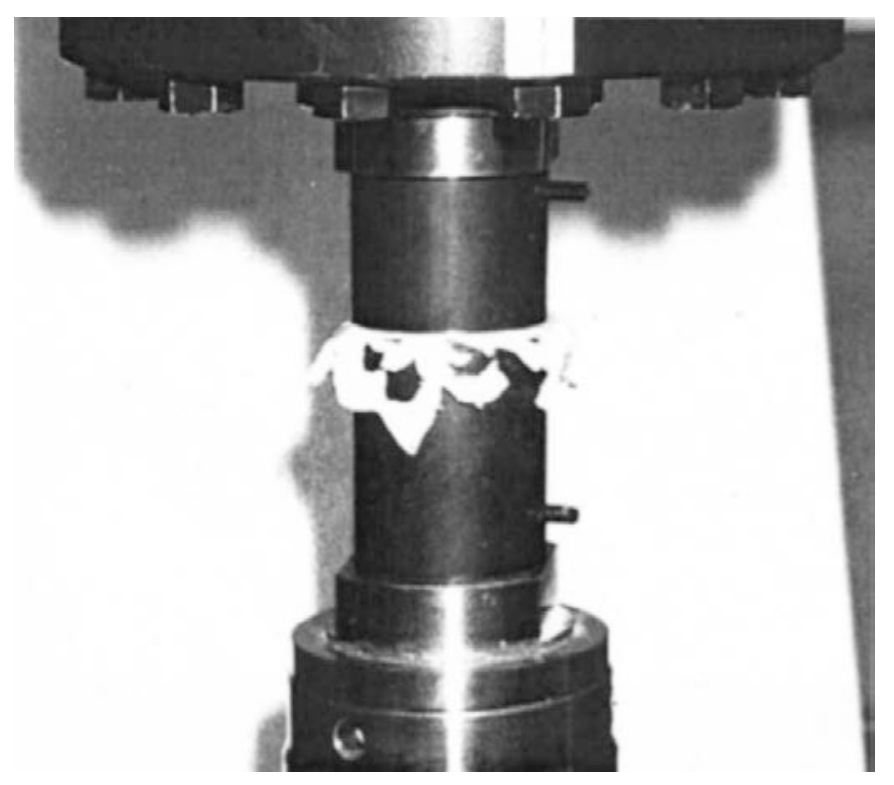

FIG. 8. The sample at the end of the test. 


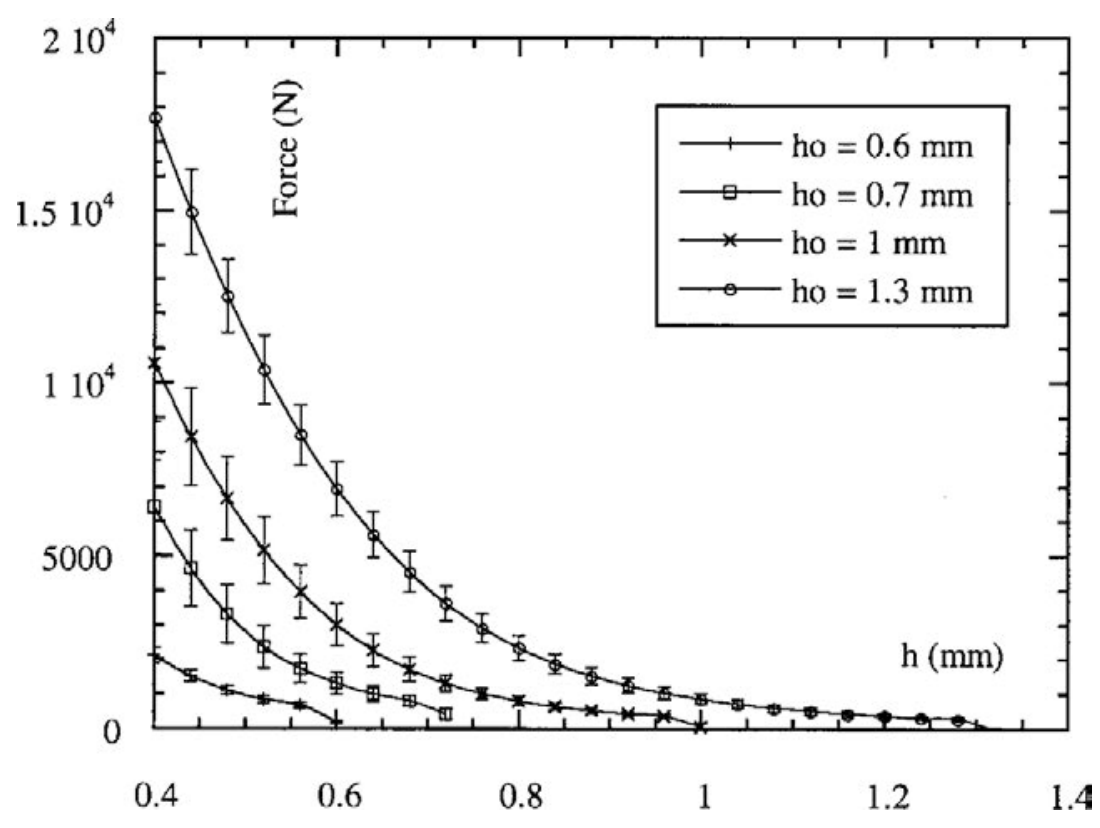

FIG. 9. Influence of the initial gap size $h_{0}$.

assumption which had already been made by many authors: Kendall [Kendall (1987)], for example, observed this type of phenomenon on cement pastes; Lanos [Lanos (1993)], on ash pastes, also observed a very strong migration of phases resulting in a solid nucleus at the center of the specimen. This reveals two interesting features of these materials:

(i) It is, generally, impossible to test them in a homogeneous manner.

(ii) However, at high velocities, the force increases with velocity, which is also an indication that a homogeneous test is possible in such a case.

\section{B. Effect of initial thickness $\boldsymbol{h}_{0}$}

Figure 9 shows results obtained at constant squeezing velocity $V=0.02 \mathrm{~mm} / \mathrm{s}$ with polished plates $20 \mathrm{~mm}$ in diameter and for different initial gap sizes $h_{0}$. These results show that for fixed gap size $h$ and velocity $V$, the measured force depends on the initial gap size $h_{0}$ and, therefore, on the strain history of the paste. If we assume that the strain can dissociate a specimen, then forming the specimen with an initial thickness $h_{0}$ has to be undertaken with great care. In particular, it is not possible to presqueeze it slowly. This is the reason why we preshaped the samples.

\section{MATHEMATICAL MODELING}

\section{A. Physical concept: existence of a critical velocity}

In our material, we can distinguish three different causes of dissipation:

(i) The intrinsic viscous dissipation of the fluid (binder) on the microscopic scale:

$$
D_{f}=\int_{\Omega} \eta_{f} \dot{\gamma}_{f}^{2} d v
$$

where $\dot{\gamma}_{f}$ is the fluid shear rate, whose dimension is $v_{f} / h$, where $h$ is the gap between plates.

(ii) The intrinsic viscous dissipation caused by friction between particles: 


$$
D_{s}=\int_{\Omega} K \dot{\gamma}_{s}^{m+1} d v \quad \text { with } \quad \dot{\gamma}_{s}=\frac{v_{s}}{h}
$$

where $\dot{\gamma}_{s}$ is the solid shear rate and $m$ the coefficient of shear rate sensitivity.

(iii) An interaction dissipation due to the viscous drag through the powder (Darcy's law):

$$
D_{i}=\int_{\Omega} k\left(v_{f}-v_{s}\right)^{2} d v
$$

We use a highly concentrated suspension (at a solid concentration of $60 \%$ per weight) and an aqueous binder whose viscosity is very low $\left(\eta_{f}=10^{-3} \mathrm{~Pa}\right.$ s). To a first approximation, it seems reasonable to neglect the intrinsic dissipation of the binder. Then, from a physical point of view, particle migration in our material results from a balance between two contradictory effects:

(i) On the one hand, particles tend to migrate toward zones where the intrinsic viscous dissipation is low, i.e., where the solid shear rate is low.

(ii) On the other hand, particle migration implies dissipation because of the viscous drag of the flow through the powder skeleton.

The result of this conflict is that migration occurs if the reduction in viscous dissipation when particles migrate towards low shear strain areas is greater than the dissipation required for filtration. It turns out that, for most pastes, the coefficient of rate sensitivity is strictly less than 1 . Moreover, the viscous dissipation has the dimension $\dot{\gamma}_{s}^{m+1}$ and the filtration dissipation has the dimension $\left(v_{s}-v_{f}\right)^{2}$ : therefore, the ratio between filtration and skeleton deformation is negligible at low squeezing velocities and large at high velocities. This indicates that at low velocities it is "worthwhile" for particles to migrate. The situation is reversed at large squeezing velocities for which filtration "costs" too much to enable migration. The limit between the two regimes occurs when filtration is no longer negligible. We evaluate an order of magnitude of the critical velocity:

$$
v_{s}-v_{f} \simeq \frac{v_{s}}{10} \quad \dot{\gamma}_{s} \simeq \frac{v_{s}}{h} \quad v_{s} \simeq V
$$

at least near the center

$$
V_{C}=100\left(\frac{K}{k h^{m+1}}\right)^{1 /(l-m)}
$$

\section{B. Mechanical three-dimensional analysis}

To extend this physical concept to three dimensions, we can model the heterogeneous behavior of a concentrated paste by describing the global behavior of the paste as the superposition of the behavior of each of its constituents. This approach is used in soil mechanics to model the behavior of drained porous media. Thus, the powder is assumed to consist of a quasirigid skeleton in which the binder can circulate [Coussy (1987)]. More recently, this approach has been used to model the behavior of concentrated paste in injection [Lantéri et al. (1991); Lantéri et al. (1996a); Dutilly (1998)] and extrusion [Casella (1997); Louge (1996)] processes. Two main approaches have been proposed in the literature to obtain macroscopic equations for multiphase media: 
(i) The first is the mixture theory, which is a generalization of continuum mechanics [Bowen et al. (1976); Poitou (1988); Lantéri et al. (1996a)]. Conservation laws are written directly for each phase on the macroscopic scale, which leads directly to the model equations.

(ii) The second is the averaging method, which consists of writing conservation laws for each constituent on the microscopic scale [Gilbert (1987); Ni and Beckerman (1991)]. These equations are then integrated with convolution products to obtain conservation laws on the macroscopic scale for each phase. On this scale, each constituent is considered to be a continuous medium.

A distinction has to be made between constituents (the binder and the granular material) on the microscopic scale and phases (solids and fluid) on the macroscopic scale. In particular, we do not determine the behavior of the granular material alone, but of a solid phase consisting of the granular material lubricated by the binder.

\section{Representative volume}

In order to be able to write macroscopic conservation laws, it is necessary to define a representative elementary volume or macroscopic point. This volume has to be large compared to the size of the solid particle, but small compared to the gap size.

\section{Conservation laws}

Under the hypothesis of the existence of a representative elementary volume, our paste can be described as the superposition of two continuous media. At each macroscopic point, both phases $c$ coexist proportionately to their volume fraction $\phi_{c}$. We designate the fluid and the solid phases, respectively, by the indexes $f$ and $s$. Then, each phase is described by:

(i) its apparent density $\rho_{c}=\phi_{c} \rho_{(c)}$, where $\rho_{(c)}$ is the density of constituent (c).

(ii) its macroscopic velocity $\underline{v}_{c}$, which can differ from the velocity of another phase.

(iii) its macroscopic stress field $\boldsymbol{\sigma}_{C}$ and strain rate $\mathbf{D}_{C}=\frac{1}{2}\left[\operatorname{grad}\left(\underline{v}_{c}\right)+\operatorname{grad}\left(\underline{v}_{c}\right)^{t}\right]$; and

(iv) its own boundary conditions.

Mass balance: In the case of a nonreactive medium, we can deduce from the global mass balance of phase $c$ the local form

$$
\frac{\partial \phi_{c} \rho_{(c)}}{\partial t}+\operatorname{div}\left(\phi_{c} \rho_{(c)} \underline{v}_{f}\right)=0 .
$$

If the constituents $(c)$ on the microscopic scale are individually incompressible, the mass balance for each phase is expressed by

$$
\frac{\partial \phi_{f}}{\partial t}+\operatorname{div}\left(\phi_{f} \underline{v}_{f}\right)=0 \quad \frac{\partial \phi_{s}}{\partial t}+\operatorname{div}\left(\phi_{s} \underline{v}_{s}\right)=0 \text {. }
$$

These equations indicate an apparent compressibility of each macroscopic phase which enables dissociation without any diffusion mechanism. Moreover, if the mixture (as is the case of our paste) is saturated (no air or gas inside), then the volume fraction verifies:

$$
\phi_{f}+\phi_{s}=1 \text {. }
$$

Momentum balance: Our paste is at a concentration of $60 \%$ per weight; its global viscosity is about $300 \mathrm{Pas}$ and the powder density is $3.35 \mathrm{~g} / \mathrm{cm}^{3}$. Mass and inertia forces 
can be neglected with respect to viscous effects. The momentum balance for each phase can thus be written as [Lantéri et al. (1991)]:

$$
\operatorname{div}\left(\sigma_{f}\right)+\underline{\pi}_{f}=\underline{0} \quad \operatorname{div}\left(\sigma_{s}\right)+\underline{\pi}_{s}=\underline{0} .
$$

Since the flow considered is isothermal, it is not necessary to write the balance of energy and entropy.

\section{Constitutive equations and boundary conditions}

Momentum supply: Assuming that the filtration is isotropic, it is generally agreed that the interaction force is the sum of a Darcy term and a reaction caused by the gradient of volume fraction:

$$
\underline{\pi}_{s}=-\underline{\pi}_{f}=k\left(\underline{v}_{f}-\underline{v}_{s}\right)+p \operatorname{grad} \phi_{s},
$$

with $\phi_{s}=1-\phi_{f}$, and $k$ being the interaction coefficient between the solid and the fluid, i.e., the ratio between the viscosity $\eta_{f}$ of the binder and the permeability $B$ of the skeleton [Costet and Sanglerat (1975)]. $B$ depends on many parameters, such as the size of the particles, their shape, their morphology, their chemical properties, etc. In order to minimize the number of adjustable parameters, we assume here that the permeability $B$ follows the Kozeny expression

$$
B=b \frac{\phi_{f}^{3}}{\left(1-\phi_{f}\right)^{2}}
$$

with

$$
b=\frac{1}{2 C T^{2} S^{2}} \simeq \frac{D^{2}}{100},
$$

where $T$ is the tortuosity of the pores, $S$ the specific surface of the powder, $C$ the circularity coefficient which defines the shape of the pores, and $D$ the diameter of the particles.

Constitutive equation for the fluid phase: The binder used in our paste is a mixture of water and nitric acid. Assuming that the binder is Newtonian and incompressible on the microscopic scale and that the particles are rigid and not in direct contact with one another, along with Gilbert (1987) we write:

$$
\boldsymbol{\sigma}_{f}=-\phi_{f} p \mathbf{I}+2 \eta_{f} \mathbf{D}_{f}, \quad \text { where } \eta_{f}=\mathrm{cst}
$$

where $\eta_{f}$ is the viscosity of the fluid and $\underline{v}_{f}$ its macroscopic velocity.

Constitutive equation of the solid phase: The macroscopic constituent referred to as the solid phase is made up of solid particles surrounded by a fine layer of adsorbed fluid. This constituent cannot be tested separately and it is impossible to measure its behavior. Therefore, we can reasonably assume, like Lantéri et al. [Lant éri et al. (1996b)], that the solid phase has purely viscous behavior which depends only on the velocity of the solid phase:

$$
\boldsymbol{\sigma}_{s}=-\phi_{s} p \mathbf{I}+2 \eta_{s} \mathbf{D}_{s} \quad \text { with } \quad \eta_{s}=K{\sqrt{2 \operatorname{tr}\left(\mathbf{D}_{s}^{2}\right)^{m}}}^{-1}
$$

where $K$ is the consistency and $m$ the coefficient of rate sensitivity. 
Many experimental and theoretical approaches [Krieger and Dougerthy (1959); Quemada (1977)] lead us to assume that the consistency $K$ diverges as the volume fraction approaches the maximum particle packing $\phi_{m}$. We extend these results to our power law behavior:

$$
K=\frac{K_{0}}{\left(1-\frac{\phi_{s}}{\phi_{m}}\right)^{\alpha}} \quad \text { with } \alpha>0,
$$

where $\alpha$ is chosen in order to be consistent with Einstein's model for the viscosity of dilute sphere suspensions:

$$
K=K_{0}\left(1+2.5 \phi_{s}\right)
$$

Then, one obtains

$$
\alpha=2.5 \phi_{m} .
$$

To a first approximation, we assumed that the maximum particle packing density $\phi_{m}$ is equal to the compact packing density of disorderly rigid spheres: $64 \%$.

Boundary conditions: We assume that the fluid and solid phases stick to the wall:

$$
\underline{v}_{f}=\underline{v}_{s}=\underline{v}_{\text {wall }} \text {. }
$$

At the outflow of the plates, the boundary condition on both phases expresses local equilibrium with the outside medium:

$$
\begin{aligned}
& \boldsymbol{\sigma}_{f} \underline{\underline{n}}=-\phi_{f} p_{a} \underline{\underline{n}} \\
& \boldsymbol{\sigma}_{s} \underline{\underline{n}}=-\phi_{s} p_{a} \underline{\underline{n}},
\end{aligned}
$$

where $\underline{n}$ is the outward normal vector and $p_{a}$ the ambient pressure.

\section{Numerical resolution}

Since the problem is unsteady, we adopted an incremental approach in three steps [Racineux (1999); Racineux et al. (1999)].

(i) In the first step, we assume that the volume fraction is known and we solve the flow problem, which is of the same level of complexity as the Stokes' problem. We use the finite element method with a velocity-pressure formulation and a Newton-Raphson algorithm to solve the nonlinearity.

(ii) Then, the volume fraction is actualized using an implicit algorithm and a TaylorGalerkin formulation by solving the equation of mass transport balance. The difficulty of the calculation results mainly from the hyperbolic nature of this equation.

(iii) At the end of a time increment the mesh is convected in order to keep the size of the elements constant.

\section{IDENTIFICATION}

\section{A. Behavior of the solid phase}

The behavior of the solid phase $(m, K)$ is identified at high velocity, i.e., in flow conditions which make the paste remain homogeneous. In this particular case, the velocity of the fluid phase is equal to the velocity of the solid phase and, therefore, the tensor of homogeneous equivalent stresses can be expressed as:

$$
\boldsymbol{\sigma}=-p \mathbf{I}+2 K \sqrt{2 \operatorname{tr}\left(\mathbf{D}^{2}\right)}(m-1) \mathbf{D}_{s} .
$$




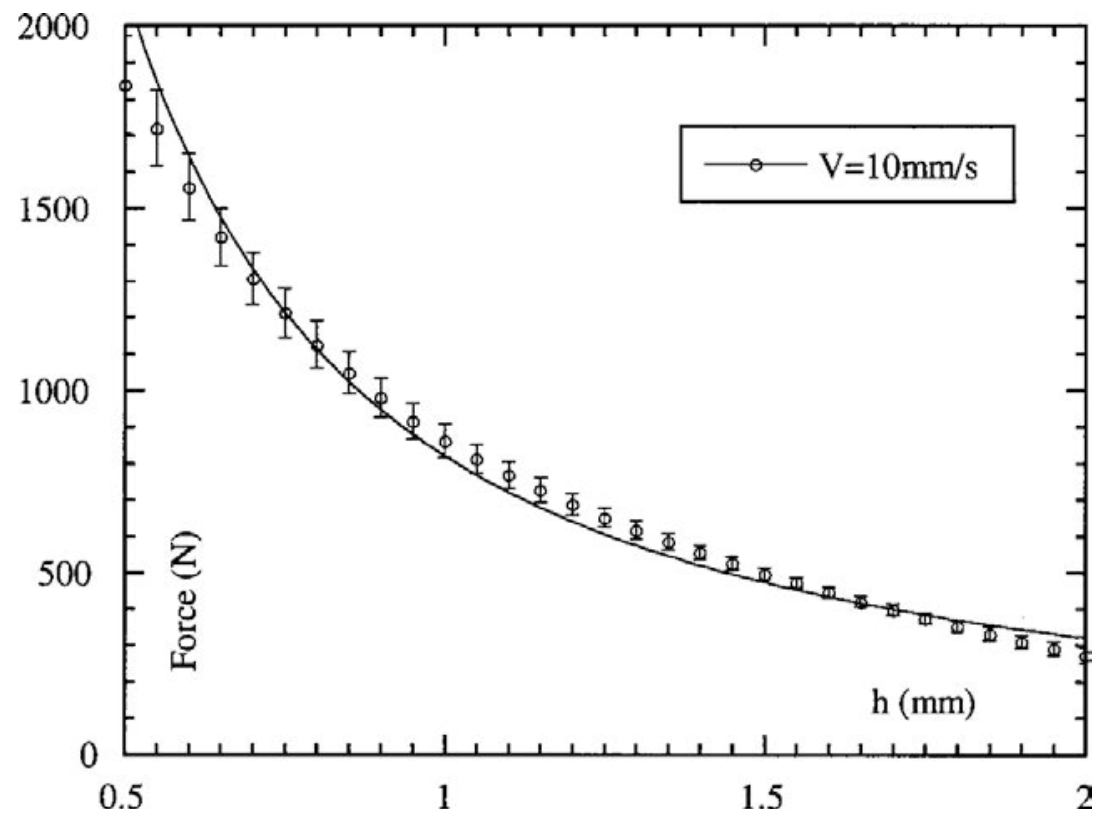

FIG. 10. The solid phase behavior is identified with a power law.

Using the Hele-Shaw approximations (since the tests were carried out for $h / R \ll 1$ ), one shows that the squeezing force is

$$
F=2 \pi\left(\frac{2 m+1}{m}\right)^{m} \frac{1}{(m+3)} \frac{K}{2^{(m-1 / 2)}} \frac{V^{m}}{h^{(2 m+1)}} R^{(m+3)} .
$$

We identified $m$ and $K$ on the force curve obtained at a compression velocity of $10 \mathrm{~mm} / \mathrm{s}$ (Fig. 10). Using a least-square method, we perform a best-fit approximation of the power law to experimental data, which leads to:

$$
m=0.01 \quad K=22600 .
$$

The coefficient of strain rate sensitivity is very close to zero, which means that the homogeneous paste behaves like a rigid-plastic material (Bingham fluid).

\section{B. Interaction coefficient}

The interaction coefficient $k$ is the ratio between the binder viscosity $\eta_{f}$ and the permeability of the skeleton $B$ :

$$
k=\frac{\eta_{f}}{B}
$$

The permeability is identified at low velocity, i.e., in a test in which dissociation actually occurs (drained test at $0.1 \mathrm{~mm} / \mathrm{s}$ ). In this case, the flow equations have no analytical solution. Therefore, we use the finite element code to simulate the squeezing test. Since we must identify only one parameter, we proceed by dichotomy and obtain the interaction coefficient:

$$
k=k_{0} \frac{\phi_{s}^{2}}{\left(1-\phi_{s}\right)^{3}}
$$

with

$$
k_{0}=5.10^{12} \mathrm{~Pa} \mathrm{~s} \mathrm{~m}^{2} \text {. }
$$




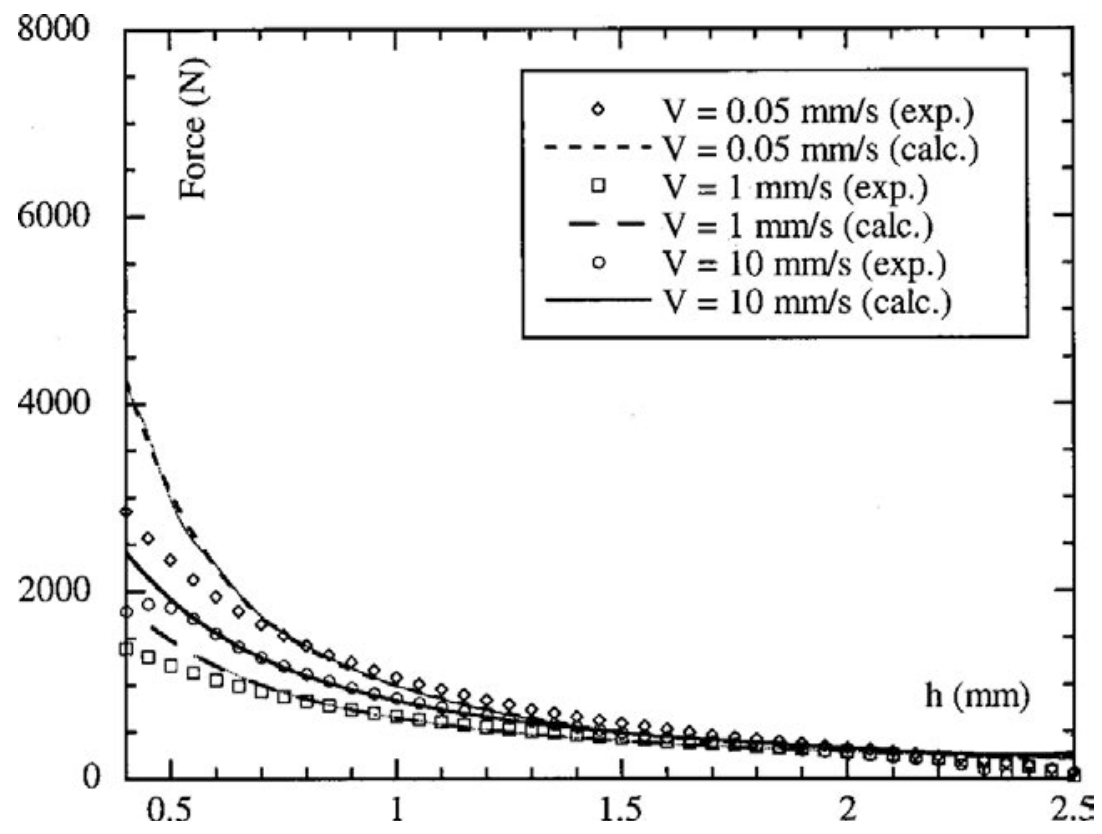

FIG. 11. Comparison between experiments and calculations.

\section{Discussion}

\section{Simulation results}

The rheological parameters were identified with two experimental curves. Figure 11 shows the superposition of experimental results and numerical simulations for the squeezing test at every compression velocity. Figure 12 shows the evolution of the volume fraction calculated with our model at a point located at the center, under the upper plate, for different compression velocities. Three remarks must be made about these curves:

(i) From Fig. 11, our model verifies the existence of paste dissociation. In particular, for velocities greater than $1 \mathrm{~mm} / \mathrm{s}$, the calculated force increases with the velocity. On the contrary, for velocities less than $1 \mathrm{~mm} / \mathrm{s}$, the force increases as the velocity decreases.

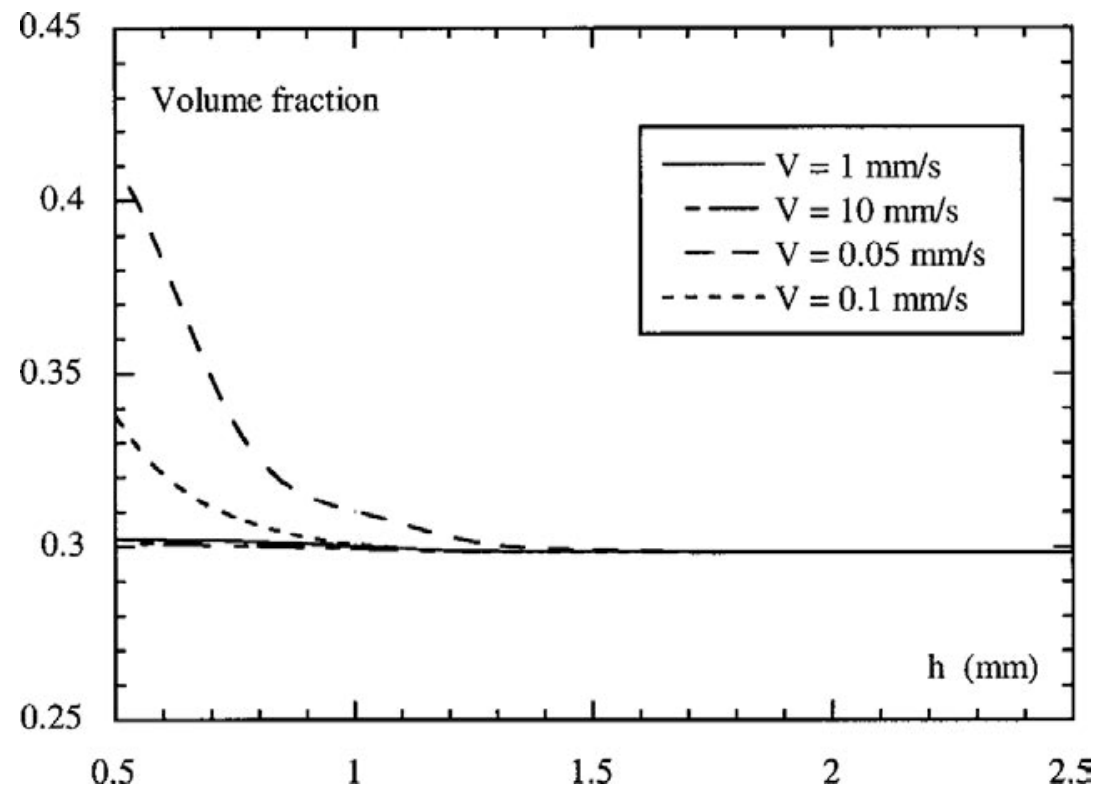

FIG. 12. Solid volume fraction calculated under the plate center. 
(ii) For high velocities (10 and $1 \mathrm{~mm} / \mathrm{s})$, the calculated dissociation is very low. This result is consistent with our strategy of identifying $K$ and $m$ at large velocities, assuming that the sample then remains homogeneous.

(iii) For low velocities, the calculated volume fractions indicate migration which is directly related to the increase of force as the velocity decreases.

Thus, there is good agreement between experimental results and simulations, at least until $h / 2$ reaches about $0.3 \mathrm{~mm}$.

\section{Order of magnitude of the interaction coefficient}

The order of magnitude of $k$ can be evaluated as a function of the viscosity $\eta_{f}$ of the binder and the permeability $B$ of the skeleton:

$$
k=\frac{\eta_{f}}{B}
$$

From relations (11), (22), and (23) one gets an estimate of $k_{0}$ as a function of the particle diameter $D$ and the fluid viscosity $\eta_{f}$ :

$$
k_{0}=\frac{100 \eta_{f}}{D^{2}}
$$

With the experimental value $k_{0}=5.10^{12} \mathrm{~Pa} \mathrm{~s} \mathrm{~m}^{2}$, this leads to a particle diameter:

$$
D \simeq 0.1 \mu \mathrm{m}
$$

This value is approximately five times larger than the particle size suggested by the mercury porosimetry measurement performed on dry samples (specific area $280 \mathrm{~m}^{2} / \mathrm{g}$ or particle size $20 \mathrm{~nm}$ ). This result, however, is in good agreement with our model if one notes that Eq. (11) holds for monodisperse granulometry only and that for polydisperse powders the larger pores have a predominant influence on permeability.

\section{Critical dissociation velocity}

From the above expression of the critical velocity [Eq. (5)] and with our experimental value for the interaction coefficient, we calculate a theoretical critical velocity $V_{C}$ $=2 \mathrm{~mm} / \mathrm{s}$ which is also consistent with the experiments (see Fig. 6). More generally, Eq. (5) combined with Eqs. (11), (22), and (23) yields

$$
V_{c}=100\left(\frac{K}{k h^{m+1}}\right)^{1 /(1-m)}=100\left(\frac{K D^{2}}{100 \eta_{f} h^{m+1}} \frac{\left(1-\phi_{s}\right)^{3}}{\phi_{s}^{2}}\right)^{1 /(1-m)} .
$$

In this expression, if the volume fraction is fixed, $m$ and $D$ are the two main materials parameters that govern the transition between the two regimes. The larger $m \in[0,1]$, the lower the critical velocity (Fig. 13). In the same way, the larger $D$, the lower $V_{C}$ (Fig. 14). From a geometrical point of view, the lower $h$, the larger $V_{c}$ (dissociation occurs more easily at the end of the test than at the beginning).

\section{Limitations}

At the end of the simulation, the results diverge greatly, particularly at low squeezing velocities. There are several possible explanations for this: the packing fraction introduced in the model could have been underestimated, which would lead to a sharp force 


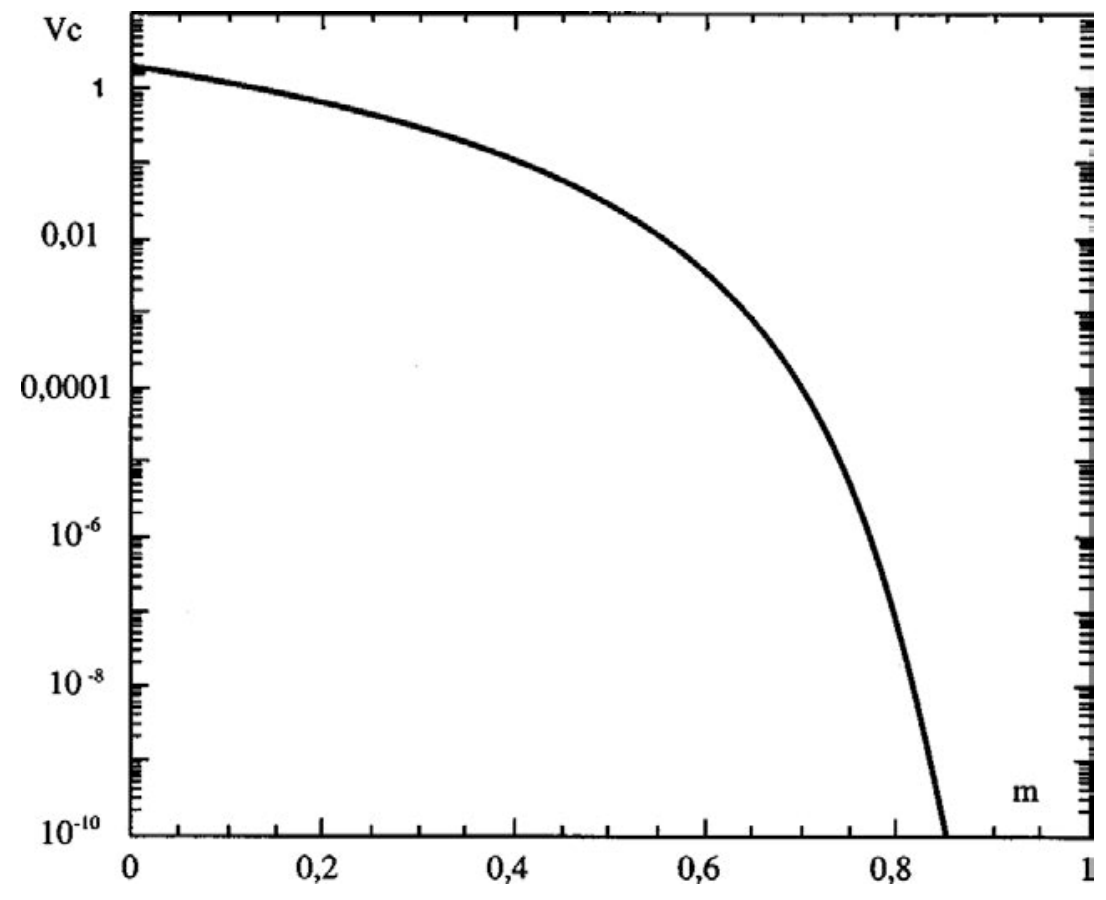

FIG. 13. Influence of $m$ on $V_{c}$.

increase when dissociation becomes important. Another explanation of the discrepancy between simulations and experiments at the end of the tests could be some numerical errors which tend to increase because of the hyperbolic nature of the mass balance equation. But, more likely, the reason is that in extending Krieger and Dougherty's relation to nonlinear viscous fluids through Eq. (14) we implicitly assumed that $m$ does not depend on $\phi_{s}$. But, as outlined above, a slight variation of $m$ can have an important impact on dissociation. It would have been possible to fit a function $m\left(\phi_{S}\right)$ in order to improve the correlation between model and experiment. However, our objective here is essentially to show that, even with only few adjustable rheological parameters (essentially $K, k, m)$, it is possible to describe this squeezing experiment quite reasonably well.

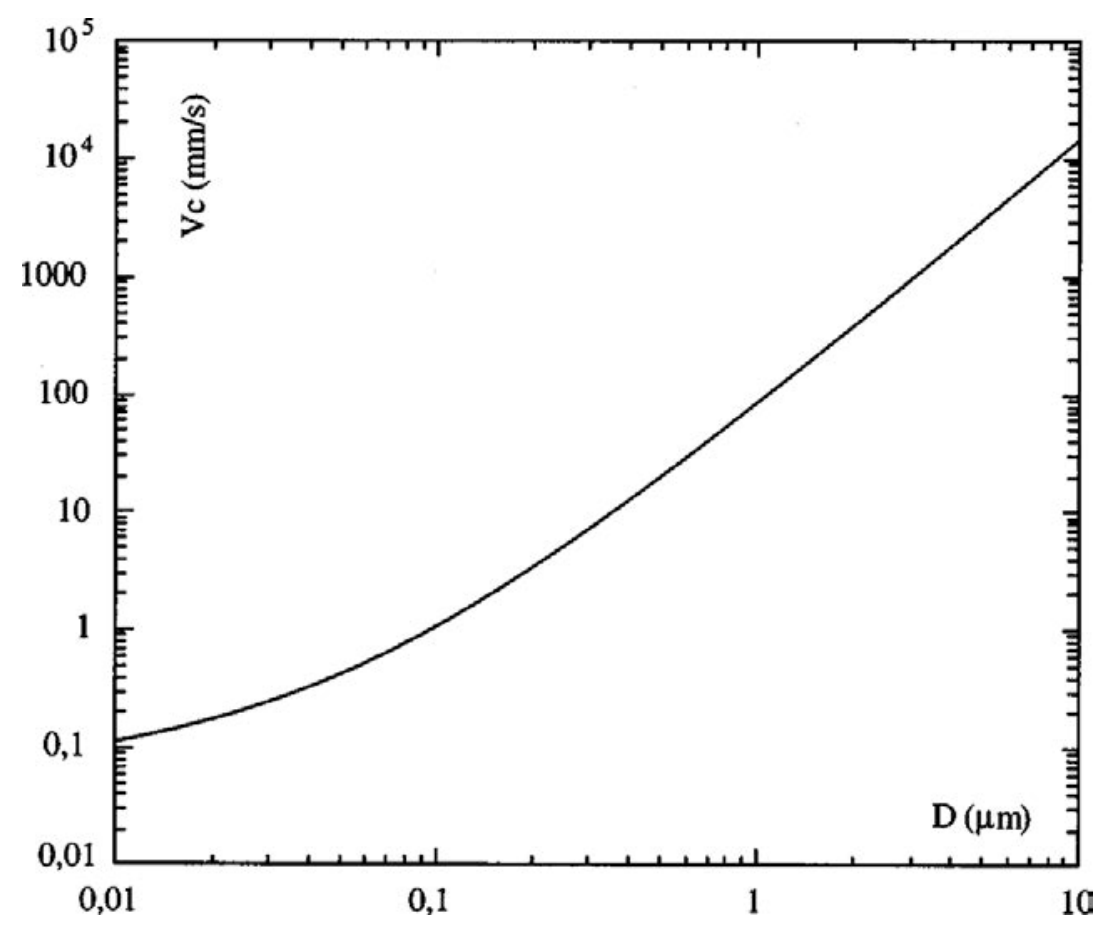

FIG. 14. Influence of $D$ on $V_{c}$. 


\section{CONCLUSION}

In this paper, we have shown indirectly that particle and binder migration occurs when a paste is deformed beyond a certain critical value of the equivalent shear strain. This phenomenon has been analyzed and modeled in the framework of the mixture theory. This theory is usually difficult to handle because of the difficulty related to the experimentation. In particular, it is a priori difficult to solicit the different phases of a heterogeneous sample separately. To cope with this difficulty, we have shown that it is possible, instead of deforming the two phases separately, to deform them either together (i.e., at large velocities) or independently (at low velocities). Thus, using a numerical program, we were able to identify the different rheological parameters of our model, which seems to lead to reasonable predictions of the overall behavior of the paste.

The paste flow can be modeled by approaches that are intermediate between nonNewtonian fluid rheology and soil mechanics. Today, the numerical difficulties of this type of model have been eliminated, at least partly, thanks to recent progress in the numerical solution of hyperbolic equations made in the last few years. Thus, mixture theory models which, a few years ago, led to difficulties in the identification of parameters are now much more practical.

\section{References}

Bowen, R. M., R. Grot, and G. A. Mauguin, Continuum Physics, Mixtures and EM Field Theories, edited by C. Eringen (Academic, New York 1976), Vol. 3.

Casella, A., "Etude du comportement d'un milieu pâteux-Approche biphasique et identification," thèse, Ecole Normale Supérieure de Cachan, 1997.

Costet, J. and G. Sanglerat, 'CCours pratique de mécanique des sols,', Dunod technique, Paris, 1975.

Coussy, O., “'Mécanique des milieux poreux,'” Technip, Paris, 1987.

Dutilly, M., “Modélisation du moulage par injection des poudres métalliques,' Thèse, Université de FrancheComté, 1998.

Gilbert, F., “Description thermo-mécanique des milieux à plusieurs constituants et application au milieux poreux saturés," Thèse, Université Paris VI, 1987.

Jabbès, S., "Mesure indirecte et directe des forces interparticulaires dans les pâtes de dioxyde de titane. Application à la mise en forme,', Thèse, Université d'Orléans, 1995.

Kendall, K., "Interparticle friction in slurries-Tribology in particulate technology,' Dunod technique, briscoe and adam edition (1987).

Krieger, I. M. and T. J. Dougerthy, "A mechanism for non-Newtonian flow in suspensions of rigid spheres," Trans. Soc. Rheol. 3, 137-152 (1959).

Lanos, C., ' 'Méthode d'identification non viscométrique de comportements de fluides,' Thèse, Institut National des Sciences Appliquées de Rennes, 1993.

Lantéri, B., H. Burlet, A. Poitou, and N. Burkarth, “Mathematical modelling for materials processing," Mark cross edition, 1991, pp. 339-356.

Lantéri, B., H. Burlet, A. Poitou, and I. Campion, "Powder injection molding: An original simulation of paste flow,'’ Eur. J. Mech. 15, 465-485 (1996b).

Lantéri, B., H. Burlet, A. Poitou, and I. Campion, "Rheological behaviour of polymer-ceramic blend used for injection moulding," J. Mater. Sci. 31, 1751-1760 (1996a).

Leighton, D. and A. Acrivos, "The shear-induced migration of particles in concentrated suspensions," J. Fluid Mech. 181, 415-439 (1987).

Louge, A., "A two phase model for paste flow including filtration and segregation effects,', C. R. Acad. Sci., Ser. IIb: Mec., Phys., Chim., Astron. 322, 785-791 (1996).

$\mathrm{Ni}$, J. and C. Beckerman, "A volume-averaged two-phase model for transport phenomena during solidification,' Metall. Trans. B 22, 349-361 (1991).

Phillips, R., R. Armstrong, R. Brown, A. Graham, and J. Abbott, "A constitutive equation for concentrated suspensions that accounts for shear-induced particle migration," Phys. Fluids 4, 30-40 (1992).

Poitou, A., “Approche mécanique du mélange par extrusion,'” Thèse, Ecole National Supérieure des Mines de Paris, 1988. 
Quemada, D., "Rheology of concentrated disperse systems and minimum energy dissipation principle-iviscosity, concentration, relationship," Rheol. Acta 16, 82-94 (1977).

Racineux, G., "Rhéologie des pâtes minérales: cas du mélange TiO2-HNO3 destiné à l'extrusion de supports de catalyseurs," Thèse, Ecole Normale Supérieure de Cachan, 1999.

Racineux, G., F. Chinesta, and A. Poitou, "Simulation de l'écoulement des pâtes minérales en vue de l'identification de leur comportement," in Proceedings of the 4th Colloque National en Calcul des Structures, Teknea, 1999, Vol. II, pp. 617-622. 